\title{
Particle-scale insight into transitional behaviour of gap-graded materials - small-strain stiffness and frequency response
}

\author{
Masahide Otsubo ${ }^{1 *}$, Troyee Tanu Dutta ${ }^{1}$, Manushak Durgalian ${ }^{2}$, Reiko Kuwano ${ }^{1}$, and Catherine O'Sullivan ${ }^{3}$ \\ ${ }^{1}$ The University of Tokyo, Institute of Industrial Science, Tokyo, Japan \\ ${ }^{2}$ Moscow State University, Faculty of Geology, Moscow, Russia \\ ${ }^{3}$ Imperial College London, Department of Civil and Environmental Engineering, UK
}

\begin{abstract}
This study aims to develop a fundamental understanding of the role of fine particles on the small-strain stiffness of gap-graded granular soils. Stiffness was measured using cyclic triaxial probes, which give a measure of static stiffness, and dynamic wave propagation data, from which the dynamic stiffness can be measured. Assemblies of loosely packed spherical particles were considered. In the laboratory, local deformation transducers were used to measure the static stiffness, while the dynamic stiffness was calculated from stress wave velocities, measured using planar piezoelectric elements. To relate the particle-scale responses to the overall soil stiffness, complementary discrete element method (DEM) simulations were performed in which both static and dynamic stiffnesses were measured. Both the laboratory and the DEM data indicate that at low fines contents $(<30 \%)$ the stiffness decreases with increasing fines content. When the fines content increases from $30 \%$ to $35 \%$ there is a sharp increase in stiffness with increasing fines content; this is understood to mark the transition point at which the fines start to contribute significantly to the overall behaviour. Analyses of the frequency domain response of shear wave signals revealed that the lowpass frequency increases significantly at this transition point. This observation can be used to develop experimental interpretation protocols to assess to what extent fines are contributing to the overall soil stiffness.
\end{abstract}

\section{Introduction}

Soil stiffness is a key parameter to predict ground deformation and it is used in earthquake site response analyses. The conventional approaches to estimate the small-strain Young's modulus $\left(E_{0}\right)$ and shear modulus $\left(G_{0}\right)$ of a granular assembly include static approaches (e.g. triaxial probes) and dynamic wave propagation approaches (e.g. resonant column, bender elements, and shear plates). The discrepancy between statically and dynamically measured small-strain stiffnesses is often noted in experimental research. Generally, $G_{0}^{d y n}$ gives a greater value than $G_{0}^{\text {sta }}$ [1] since $G_{0}$ decreases with increasing the strain level, and the dynamic approach gives the stiffness at smaller strains [2]. Discrete Element Method (DEM) simulations provide supplementary particle-scale insights into the overall stiffness and enable precise measurements of the static/dynamic stiffness [3].

The mechanical response of gap-graded soils has been considered in a number of experimental studies. The ratio of the median particle diameters $\left(D_{50}\right)$ of the coarse and fine particles $(\chi)$ and the fines context $(F c=$ mass of fines/total mass) both determine the behaviour [4]. Yang \& Liu [5] used mixtures of Toyoura sand and crushed silica fine $(\chi \approx 4)$ and found that $G_{0}{ }^{d y n}$ decreases as $F c$ increases up to $30 \%$. They prepared the mixtures maintaining an equivalent range of global void ratios (e). However, at high $\chi$ values, it is difficult to maintain a constant $e$ while varying Fc [6]. Furthermore, for laboratory tests, material segregation is inevitable at high $\chi$ values. The intergranular void ratio (or skeleton void ratio $\left.\left(e_{g}\right)[7]\right)$, and equivalent granular void ratio $\left(e_{g} *\right)$ [89] are often used to analyse the density effect on $G_{0}$. Goudarzy et al. [10] found a good correlation between $e_{g}{ }^{*}$ and $G_{0}{ }^{d y n}$ for mixtures of large and small spherical glass beads $(\chi=10)$ at $F c \leq 50 \%$. They observed that $G_{0}{ }^{d y n}$ decreases with increasing fines at $F c \leq 30 \%$; however, $G_{0}^{d y n}$ increases at $40 \% \leq F c \leq 50 \%$.

Building on experimental work focussed on internal erosion susceptibility [11], Shire et al. [12] assessed the role of fines in stress transfer using DEM and found that, for the $\chi$ values they considered, the fines just fill the voids between the coarse particles at a fines content between $24 \%$ and $29 \%$ depending on the relative density. Irrespective of density, fines begin to separate the coarse particles from one another at void ratios exceeding $35 \%$.

This contribution assesses the role of fines on the small-strain stiffness of loosely packed gap-graded materials adopting both experimental and DEM approaches. $F_{C}$ is systematically increased and the effect on both the measured stiffness and the frequency content of the received signals in the dynamic wave propagation tests and simulations is considered.

\footnotetext{
* Corresponding author: otsubo@iiis.u-tokyo.ac.jp
} 


\section{Laboratory testing}

\subsection{Materials and sample preparation}

Alkaline glass beads were used in this study so that particle shape effects could be removed from the study. The material specific density is 2.5 , the Young's modulus is $71.6 \mathrm{GPa}$ and the Poisson's ratio is 0.23 , (data from the supplier data sheet). Two distinct sizes of glass beads were used (Fig. 1(a)): $1.4-2.2 \mathrm{~mm}$ (referred to here as the coarse particles) and $0.15-0.25 \mathrm{~mm}$ (fine particles) so that $\chi \approx 8.5$. Four fines content values $(F c=0 \%, 30 \%, 40 \%$ and $50 \%$, referred to here as $F c 0$, $F c 30, F c 40$ and $F c 50$, respectively) were considered in the experimental work.

The dry mixed material was poured into a mould using a funnel keeping the elevation of its tip just above the sample surface to prepare a loose packing. The resultant void ratios for $F c 0$ and $F c 30$ were 0.658 and 0.381 , respectively. Side tapping was applied to specimens for $F c 40$ and $F c 50$ after the material was poured up to the top surface to adjust the $e$ value to be the same as that for $F c 30$. During the sample preparation, material segregation was observed at $F c 30$ and $F c 40$, and it was more marked at $F c 30$, while $F c 50$ exhibited minor segregation.

\subsection{Test procedures}

Cylindrical specimens, approximately $75 \mathrm{~mm}$ in diameter and $150 \mathrm{~mm}$ high, were prepared (Fig. 1(b)), and an external displacement transducer, two local (vertical) deformation transducers (LDTs) and three laterial deformation transducers were placed at a negative vaccum pressure of $30 \mathrm{kPa}$. The specimens were then subjected to an isotropic confinment of 100 $\mathrm{kPa}$ pneumatically and tested in dry conditions.

Prior to applying small-amplitude cyclic triaxial probes, dynamic wave propagation tests were conducted using disk transducers (shear plates) mounted inside the topcap and bottom pedestal [13-14]. A sinusoidal pulse having a frequency of $10 \mathrm{kHz}$ was inserted using a bipolar power supply; a double amplitude of $140 \mathrm{~V}$ was inserted to the transmitter. Both P- and S-wave signals were analysed to evaluate $E_{0}{ }^{d y n}$ and $G_{0}{ }^{d y n}$ as well as the frequency domain responses. a)

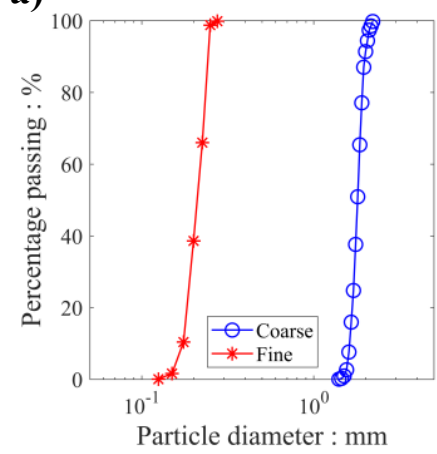

b)

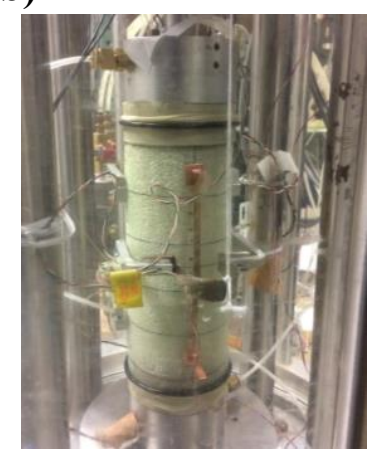

Fig. 1. (a) Particle size distribution of coarse and fine glass beads (b) Laboratory specimen.

\section{DEM simulation method}

The DEM simulations were performed using a modified version of the open-source code Granular LAMMPS [15] on the Oakforest-PACS system in the Joint Centre for Advanced High-Performance Computing (a facility shared between the Universities of Tokyo and Tsukuba, Japan). A simplified Hertz-Mindlin contact model was used considering the material properties of the alkane glass beads. Two distinct sizes of spherical particles (1.2 $-2.2 \mathrm{~mm}$ and $0.15-0.25 \mathrm{~mm}$ ) were used. The $F c$ value was varied from 0 to $60 \%$. Examples of representative packings for a middle core $(10 \times 10 \times 10 \mathrm{~mm})$ of each sample type are illustrated in Fig. 2.

Initially, non-contacting particles were created in a cubic space, and then a servo-controlled algorithm was used to isotropically compress the specimens to a mean effective pressure $p^{\prime}$ of $100 \mathrm{kPa}$. The inter-particle friction coefficient $(\mu)$ of 0.25 was used to prepare relatively loose packing. A mixed boundary of rigid walls in the vertical (axial) direction and periodic boundaries in the horizontal (lateral) direction was used. The same material properties as glass beads were considered for the rigid walls. The length of specimens varied between $131.9 \mathrm{~mm}$ and $154.2 \mathrm{~mm}$; the lateral dimensions were shorter $(11.3 \mathrm{~mm}$ to $22.0 \mathrm{~mm})$ as periodic boundaries were adopted. Since no gravitational force was included, material segregation was not observed in the DEM simulations (Fig. 2).
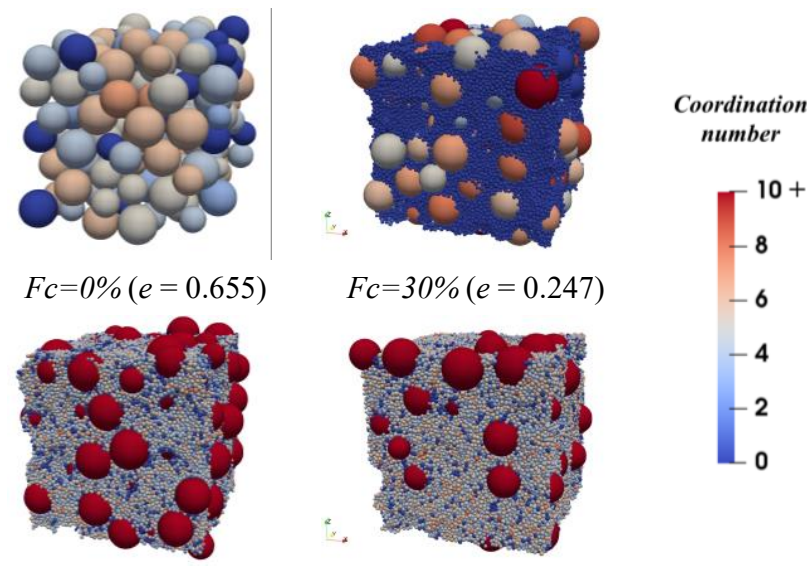

$F_{c}=40 \%(e=0.323)$

$F_{C}=60 \%(e=0.428)$

Fig. 2. Representative packing of DEM sample (middle core).

Once the specimen had achieved the target stress state, the $\mu$ value was increased to 0.5 to suppress slippage. Following the laboratory test scenario, smallamplitude cyclic triaxial probes were performed to measure $E_{0}{ }^{\text {sta }}$ and $G_{0}{ }^{\text {sta }}$, and P- and S-wave propagation simulations were conducted by perturbating the transmitter (bottom) wall horizontally and vertically, respectively [16-17]. A sinusoidal pulse with a double amplitude of $5 \mathrm{~nm}$ and a frequency of $10 \mathrm{kHz}$ was adopted. The resultant changes in shear stress and normal stress on both transmitter and receiver walls were recorded to determine the $\mathrm{P}$ - and $\mathrm{S}$-wave velocities, respectively. No damping was applied during the wave propagation simulations. 


\section{Results}

\subsection{Small-amplitude cyclic triaxial probes}

Small-amplitude cyclic triaxial probes were performed where the deviatoric stress was controlled to be approx. $\pm 1 \mathrm{kPa}$, while keeping the minor principal stress at 100 $\mathrm{kPa}$. Referring to Fig. 3, the slope of deviatoric stress axial strain relationship gives $E_{0}{ }^{\text {sta }}$. Both the laboratory and the DEM data show that $F_{c} 40$ gives the largest value of $E_{0}{ }^{\text {sta }}$, while $F c 30$ exhibits the lowest. Since the laboratory specimens $F c 30$ and $F c 40$ had a similar $e$, a sharp increase in $E_{0}{ }^{\text {sta }}$ from $F c 30$ to $F c 40$ is remarkable. Similarly, for the DEM data, Fc40 had a larger $e$; however, $E_{0}{ }^{\text {sta }}$ was considerably larger than that of $F c 30$. Discrepancies between the laboratory and DEM data are probably due to differences in $e$ and material segregation; the heterogeneous packing in the laboratory specimens was noted above.
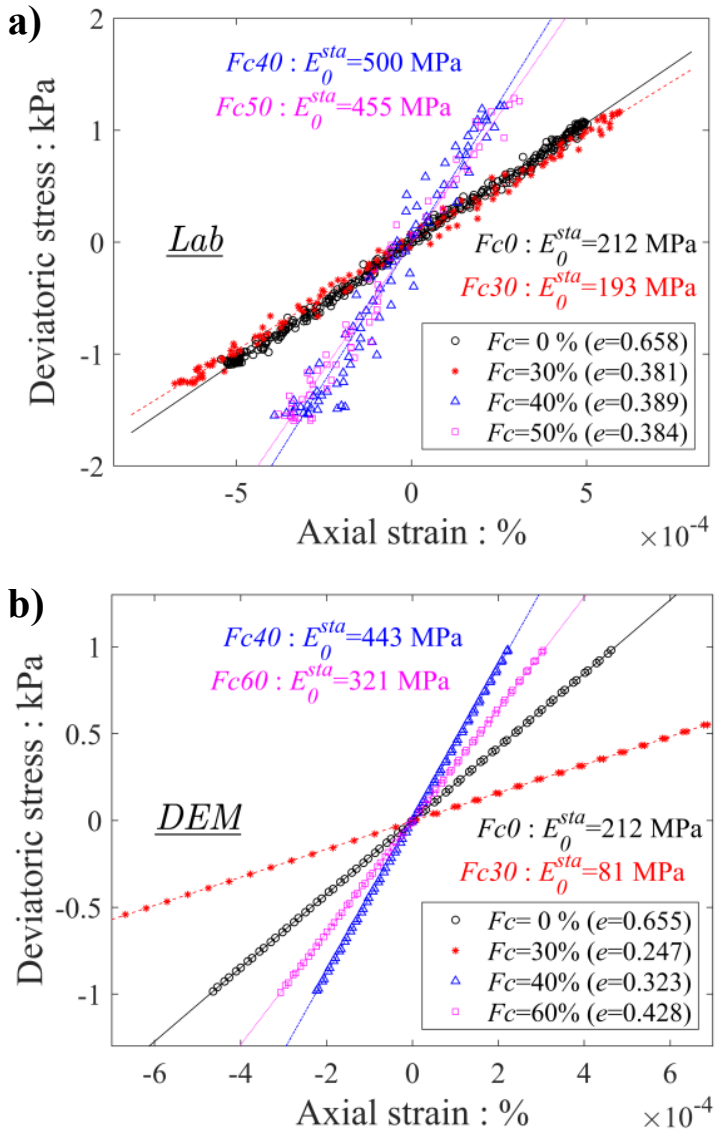

Fig. 3. Small-amplitude stress-strain relationship with varying fines content (a) Laboratory experiments (b) DEM simulations.

\subsection{Dynamic wave propagation tests}

In the dynamic tests $\mathrm{P}$ - and S-wave measurements were conducted, and the responses recorded at the transmitter and receiver plates are plotted in Fig. 4 where the horizontal axis is the time normalized by the travel distance $(L)$ to compare the arrival time of waves. For the DEM data, the transmitter responses slightly differ for the P- and S-waves, and Fig. 4(b) shows the first cycle of the P-wave response. Following Dutta et al. [14], the initial rise of P-wave signals and the peak of the first cycle of S-wave signals are both highlighted on Fig. 4. The variation in P-wave arrival times with $F_{C}$ was similar for the simulations and experiments. For the Fc30 DEM specimen, the $\mathrm{S}$-waves travelled at a considerably slower velocity than the other cases and the amplitude of the received signal was also significantly reduced. Limited S-wave data are presented for Fc50 (Lab) due to an experimental issue.
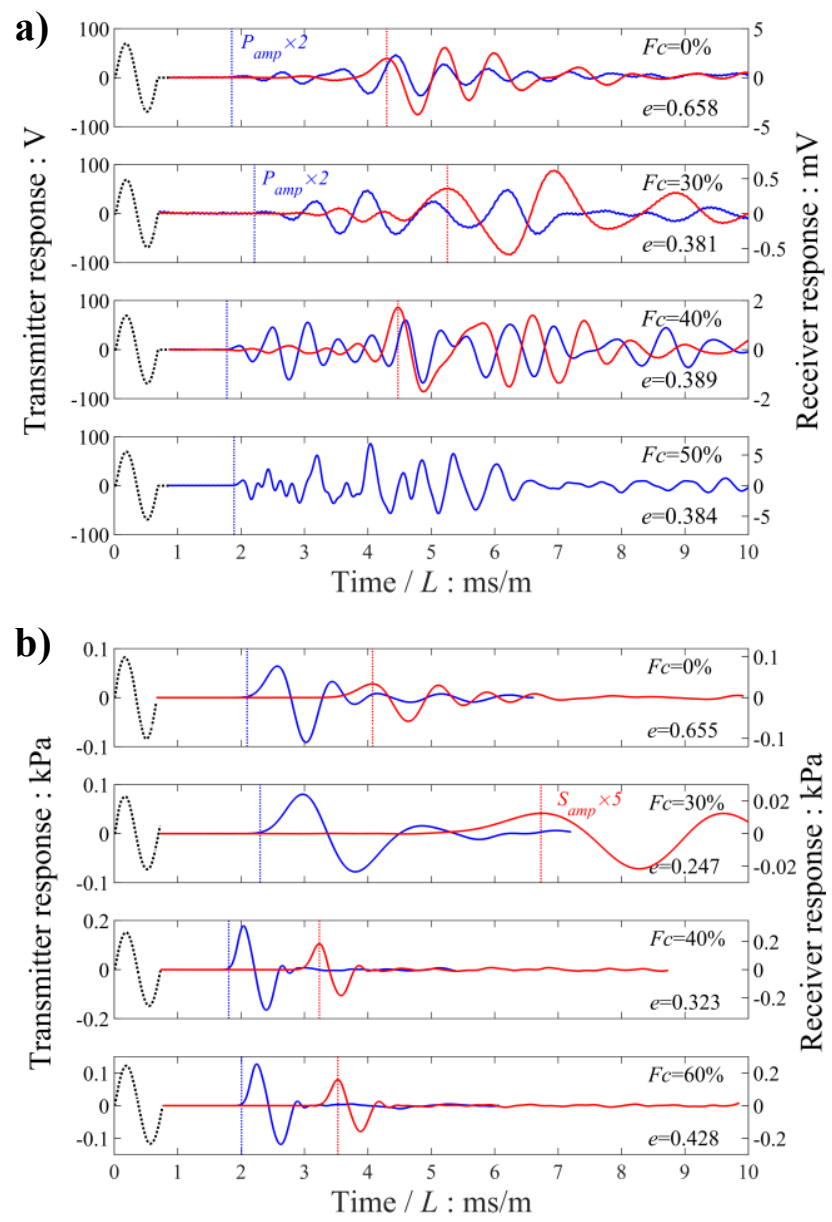

Fig. 4. Time domain responses (a) laboratory data (b) DEM data. Blue lines give $\mathrm{P}$-wave data, while red lines give $\mathrm{S}$-wave data. (Horizontal axis is time normalized by travel distance).

\section{Discussion}

\subsection{Small-strain stiffness}

The following equations were used to calculate the elastic parameters for the dynamic wave propagation tests.

$$
\begin{gathered}
G_{0}{ }^{d y n}=\gamma_{S} /(1+e) V_{S}^{2} \\
v_{0}^{d y n}=0.5\left(V_{p}^{2}-2 V_{S}^{2}\right) /\left(V_{p}^{2}-V_{S}^{2}\right) \\
E_{0}^{d y n}=2\left(1+v_{0}^{d y n}\right) G_{0}{ }^{d y n}
\end{gathered}
$$

where $\gamma_{S}=$ density of glass beads $\left(\mathrm{kg} / \mathrm{m}^{3}\right) ; V_{S}$ and $V_{p}=\mathrm{S}$ and P-wave velocities; $v_{0}{ }^{d y n}=$ Poisson's ratio. The 
resultant $E_{0}{ }^{d y n}$ and $G_{0}{ }^{d y n}$ values as well as the static equivalent values are compared in Fig. 5 where a void ratio correction function for spherical particles $[14,17]$ was adopted as:

$$
f e=(1.28-e)^{2} /(1+e)
$$

Equation 4 considers the global void ratio, rather than using $e_{g}$ [7] or $e_{g}{ }^{*}$ [8-9]).

The laboratory data follow the overall trend observed for the DEM data considering both $E_{0}$ and $G_{0}$; both stiffness values decrease with increasing $F_{C}$ up to $30 \%$ followed by a sudden increase at $30 \%<F c<35 \%$. For the DEM data, the dynamic stiffness is consistently greater than the static stiffness. Supplementary data (not shown here) exhibit a close match between $G_{0}{ }^{d y n}$ and $G_{0}{ }^{\text {sta }}$ for denser packings. However, for the laboratory specimens, a good agreement between the static and dynamic stiffnesses was observed for $F_{c} 0$ and $F_{c} 30$, but not for Fc40. This discrepancy is probably due to segregation of materials at the transitional fines content.
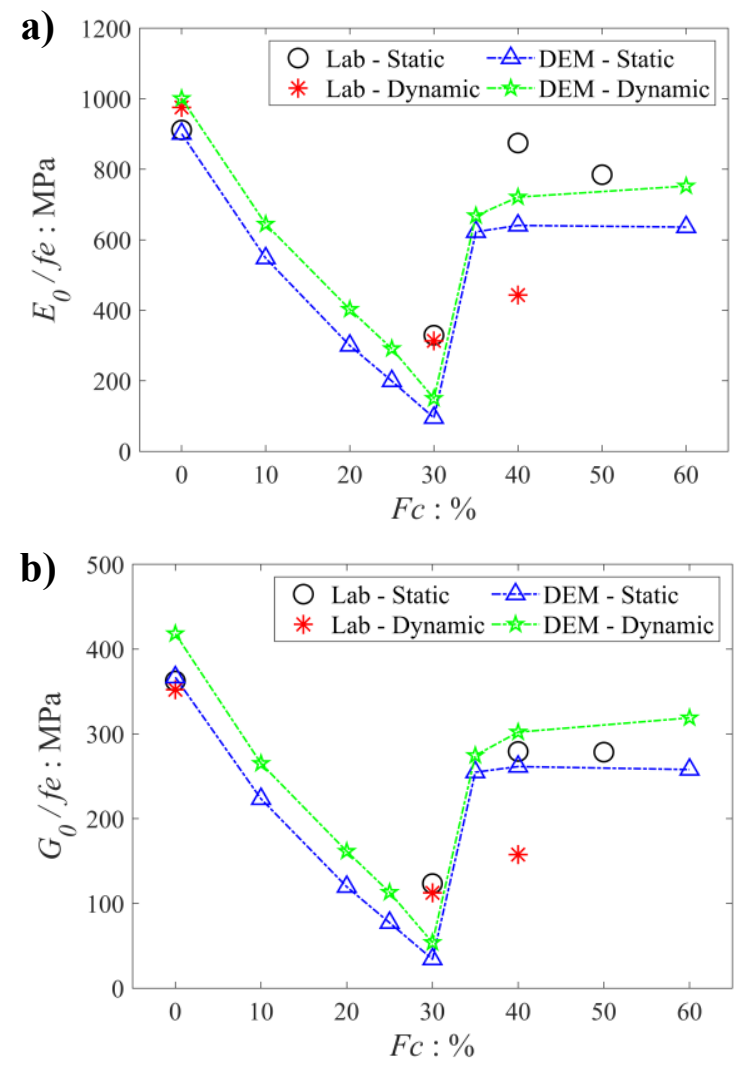

Fig. 5. Variation in (a) $E_{0} / f e$ (b) $G_{0} / f e$ with fines content.

\subsection{Contribution of fines}

Referring to Fig. 2, most of the fines in the DEM sample of $F c 30$ are not in contact with surrounding particles (underfilled fabric), while the fines for Fc40 and Fc60 are in contact, indicating that fines carry stresses [12] and contribute to the overall stiffness (overfilled fabric). The distribution of three types of mechanical coordination numbers (i.e. excluding particles having 0 or 1 contact) with varying $F c$ is illustrated in Fig. 6 . The coarse-coarse contact type is in the majority at $F c \leq$
$30 \%$, while the fine-fine contact type increases sharply between $30 \%<F c<35 \%$. The coarse-fine contact type per fine particle is 2 or less due to the large size ratio $(\chi$ $\approx 8.5$ ) which prohibits a fine particle simultaneously contacting three coarse particles. This sudden change highlights the onset of the overfilled fabric and at this state the material becomes stiffer as seen in Fig. 5 .

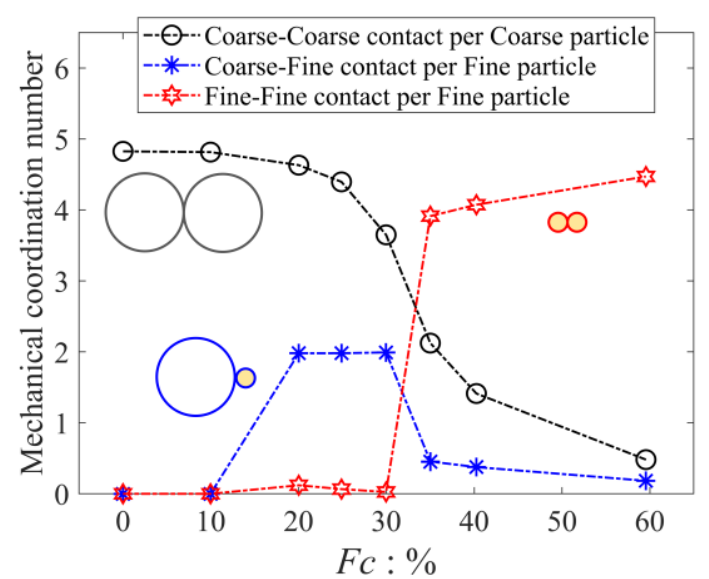

Fig. 6. Distribution of three types of mechanical coordination numbers with varying fines content.

\subsection{Frequency domain response}

Dutta et al. [14] showed that the lowpass frequency $\left(f_{l p}\right)$ for $\mathrm{S}$-waves increases as particle size decreases $\left(f_{l p} \propto\right.$ $\left.1 / D_{50}\right)$. Here, the sensitivity of $f_{l p}$ to $F c$ is illustrated in Fig. 7. A sharp rise in $f_{l p}$ is evident between $F_{c} 30$ and Fc35; indicating that the change in the fine particles' contribution to the overall behaviour influences the dynamic wave propagation response. This observation is well correlated with the variation in both $E_{0}$ and $G_{0}$ (Fig. 5 ) as well as the coordination number distribution (Fig. 6 ). This result indicates that quantifying $f_{l p}$ enables the extent to which fine particles into the overall stiffness be evaluated using geophysics laboratory tests. Recalling that this study considered relatively loose samples, the transitional $F C$ value becomes lower for denser samples [12]. The $f_{l p}$ value depends on packing density [16], whose effects on Fig. 7 should also be analysed further.

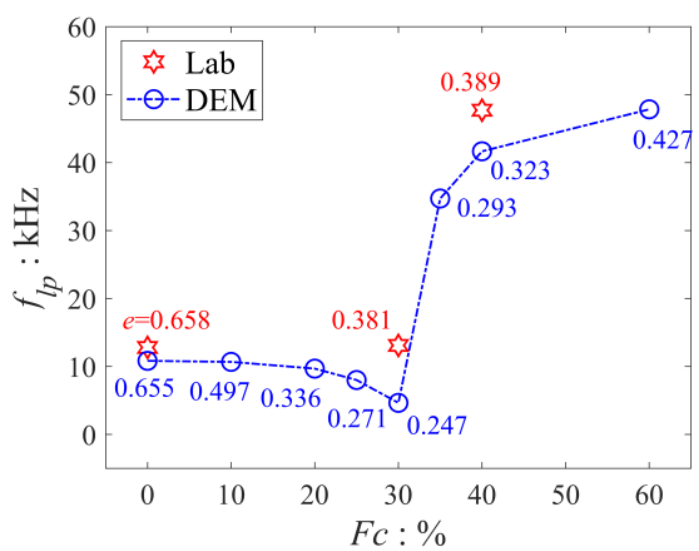

Fig. 7. Variation in lowpass frequency $\left(f_{l p}\right)$ with $F_{c}$ (void ratio value is shown for each test case). 


\section{Conclusions}

This contribution has assessed the influence of fines content $(F C)$ on the small-strain stiffness of loosely packed gap-graded materials considering both static and dynamic testing approaches. Laboratory experimental results and DEM simulation results concurred so that the following conclusions can be made:

- For the samples comprising spherical particles with $\chi=8.5$ considered here, at low fines contents $\left(F_{C}<30 \%\right)$ where the material is underfilled, $E_{0}$ and $G_{0}$ decrease with increasing $F_{C}$ even though the global void ratio decreases.

- A transitional behaviour was observed at $30 \%<F_{c}<35 \%$; over this range $E_{0}$ and $G_{0}$ increase sharply indicating that the fines start to participate in the overall mechanical behaviour, this marks the onset of an overfilled fabric.

- The DEM analysis of coordination number revealed that the fine-fine contact type increases sharply at $30 \%<F c<35 \%$ for the relatively loose packing considered in this study.

- The contribution of the fines to the overall stiffness can be evaluated by assessing $f_{l p}$ based on geophysics dynamic wave measurements.

- Dynamic stiffness was greater than static stiffness for DEM data. Better agreement was seen for the laboratory data; however, segregation affects the accuracy of measurement in the laboratory.

A part of this research was based on the Oakforest-PACS system operated at the Joint Center for Advanced HPC (JCAHPC) under cooperation with the Information Technology Center at the University of Tokyo and the Center for Computational Sciences at the University of Tsukuba.

\section{References}

1. L.P. Suwal, R. Kuwano, Geotechnical Testing Journal 36(4), 493-505 (2013)

2. C.R.I. Clayton, Géotechnique 61(1) 5-37 (2011)

3. O. Mouraille, W.A. Mulder, S. Luding, J. Stat. Mech. P07023 (2006)

4. T. Shire, C. O’Sullivan, K.J. Hanley, Granular Matter 18:52 (2016)

5. J. Yang, X. Liu, Géotechnique 66(6), 500-514 (2016)

6. P.V. Lade, C.D. Jr. Liggio, J.A. Yamamuro, Geotechnical Testing Journal 21(4), 336-347 (1998)

7. R. Salgado, P. Bandini, A. Karim, J. Geotech. Geoenviron. Eng. 126(5), 451-462 (2000)

8. S. Thevanayagam, T. Shenthan, S. Mohan, J. Liang, J. Geotech. Geoenviron. Eng. 128(10), 849-859 (2002)

9. M.M. Rahman, S.R. Lo, C.T. Gnanendran, Can. Geotech. J. 45(10), 1439-1456 (2008)
10. M. Goudarzy, D. König, T. Schanz, Soils and Foundations 56(5), 756-764 (2016)

11. A.W. Skempton, J.M. Brogan, Géotechnique 44(3), 449-460 (1994)

12. T. Shire, C. O’Sullivan, K.J. Hanley, R.J. Fannin, J. Geotech. Geoenviron. Eng. 141(12) (2015)

13. L.P. Suwal, R. Kuwano, Soils and Foundations 53(4), 510-524 (2013).

14. T.T. Dutta, M. Otsubo, R. Kuwano, C. O’Sullivan, Géotechnique Letters (2019) (under review)

15. S. Plimpton, J. Comput. Phys. 117(1), 1-19 (1995)

16. M. Otsubo, C. O’Sullivan, K.J. Hanley, W. Sim, Géotechnique 67(5), 452-459 (2017)

17. M. Otsubo, C. O'Sullivan, Soils and Foundations 58(3), 602-614 (2018). 